\title{
The impact of the Covid-19 pandemic on maternal delivery experiences and breastfeeding practices in China: data from a cross-sectional study
}

\author{
Jinyue Yu ${ }^{1 *+}$, Mingyue Gao ${ }^{1 \dagger}$, Zhuang Wei $^{2}$, Jonathan C. K. Wells ${ }^{1}$ and Mary Fewtrell ${ }^{1}$
}

\begin{abstract}
Background: The COVID-2019 pandemic has placed extensive pressure on health systems and posed a severe public health challenge worldwide. Lockdown measures implemented in many countries have delayed virus spread. However, a considerable number of people have faced unprecedented pressure, especially pregnant and breast-feeding women, because face-to-face professional support has been reduced during the lockdown in many countries.
\end{abstract}

Objectives: To compare the delivery and infant feeding experiences of women who delivered before (BL) versus during (DL) the Covid-19 pandemic in Beijing, China and to investigate predictors of breastfeeding at 6-months.

Methods: Women aged $\geq 18$ years with an infant $\leq 18$ months of age completed an anonymous survey. Information/links were shared online and via local clinics in Beijing. Logistic regression was performed to assess predictors of breastfeeding during the first 6 -months.

Results: One thousand eight hundred seven women provided data; BL 1231 (68.1\%), DL 576 (31.9\%). Significantly more mothers in DL group reported the lockdown had moderate to high impact to their household income ( $p=0.013$ ) and the convenience of purchasing daily necessities $(p=0.014)$. Compared to BL mothers, significantly more mothers in the DL groups thought their birth location and breastfeeding intention had been effected by the COVID-19 ( $p<0.001, p=0.036$ respectively). Mostly breastfeeding (MBF, mainly breastfeeding with few non-formula fluids added) at 6 months was predicted by delivery during the lockdown period (OR1.43, 95\% confidence interval (Cl) $1.08,1.90)$, younger maternal age (OR $0.96,95 \% \mathrm{Cl} 0.93,0.99)$, getting support from friends or relatives $(\mathrm{OR} 1.95,95 \% \mathrm{Cl}$ 1.06, 3.59), and discussing health issues in online groups > four times a week (OR 1.66, 95\% Cl 1.09, 2.53).

Conclusion: The COVID-19 pandemic and lockdown measures influenced mothers' planned birth location and breastfeeding intention. However, breastfeeding practice was maintained during the pandemic. Our results highlight the importance of feeding support as well as potential beneficial effects of increased mother-infant contact during the lockdown period which is relevant even under normal circumstances.

Keywords: COVID-19, Birth, Infant feeding, Postpartum, Breastfeeding

${ }^{*}$ Correspondence: jin.yu.16@ucl.ac.uk

${ }^{\dagger}$ Jinyue Yu and Mingyue Gao contributed equally to this article.

${ }^{1}$ Population, Policy \& Practice Research \& Teaching Department, UCL

Institute of Child Health, University College London, London, UK

Full list of author information is available at the end of the article

\section{Synopsis}

Study question: What impact did the pandemic have on delivery experience and infant feeding and are there practical measures that can be identified to improve maternal experiences and infant feeding? 
What's already known: Previous studies showed breastfeeding can be affected by changes of maternal social-economic status, supports, and delivery experiences that influenced by the COVID-19 pandemic, and the impact may vary among countries.

What this study adds: The study assessed the relationship between lockdown measures during the COVID-19 pandemic and their impacts on maternal delivery and feeding practice in Beijing, China. Results suggested that the CPVID-19 pandemic had affected mothers' planned birth location and breastfeeding for the first 6 months of birth. Notably, we found mothers with younger age, getting support from friends or relatives compared to no support, and chatting in online maternal supporting group more than 4 times a week were more likely to breastfeeding their infants.

\section{Introduction}

The coronavirus disease 2019 (COVID-2019) pandemic, caused by severe acute respiratory syndrome coronavirus 2 (SARS-CoV-2), began in December 2019 and has placed extensive pressure on health systems and posed a severe public health challenge worldwide [1, 2]. COVID-19 affects people of all ages, with no significant gender difference [3-5]. Lockdown measures, including closure of schools, workplaces and other public places, were implemented in many countries and have delayed virus spread [6]. Nevertheless, confronted with the COVID-19 pandemic and pervasive disruption to daily life,many people have faced unprecedented pressure. This is particularly the case for pregnant and breast-feeding women, because face-to-face professional support has been reduced during the lockdown in many countries.

Breast milk is the optimum food for infants, and provides the energy and essential nutrients required during the first few months of life as well as non-nutritive bioactive components, many of which provide protection against infection [2]. A systematic review including 49 studies found no association between infection of COVID-19 and feeding method or maternal proximity [7]. The World Health Organization (WHO) also recommended that mothers with COVID-19 (or suspected COVID-19) should breastfeed as long as they take appropriate precautions $[4,8]$. However, the lockdown measures undertaken in several countries to prevent the spread of virus impacted maternal postpartum experiences, leading to distress for a large number of women and affecting their breastfeeding practice [9-11].

In Beijing, China, general lockdown measures were in place between from 26th January to 31st July 2020 [12]; most public places apart from essential stores were closed. Travel restrictions were imposed and meeting with those from other households limited, other than for caring or work purposes. Additionally, as a means of ensuring the safety of mothers and babies, routine postpartum home visits were replaced with telephone consultations, although mothers were able to request face-to-face appointments if required, following a triage process to identify suspected cases of COVID-19. Although these strategies effectively protected postpartum women from infection, the impact of such strict policies on women's wellbeing and breastfeeding practice remains unknown. Studies show that postnatal experiences and breastfeeding support are strongly associated with breastfeeding initiation and duration [13-15]. Given that breastfeeding is optimally supported through high-quality professional and peer-to-peer support and positive maternal wellbeing [16], and the COVID-19 pandemic is ongoing in a large majority of countries, it is important to understand the impact of the pandemic and lockdown measures on women's ability to breastfeed.

This study therefore aimed to investigate: 1 ) The delivery experience, feeding intentions and actual feeding methods of mothers who delivered before or during the lockdown; 2) The impact of lockdown on breastfeeding practice and support; 3 ) Factors that predict infant feeding methods in the first 6 months. The research is important to identify problems experienced by this vulnerable group, and will also contribute to the formulation of public health policies as the current COVID-19 pandemic continue, as well as in future public health emergencies.

\section{Methods \\ Recruitment}

The research was undertaken between 1st August 2020 and 31st October 2020, at a number of local clinics attached to Beijing Children's Hospital $(\mathrm{BCH})$ and located in different districts in Beijing, China. Advertisements, including a brief introduction to the research and the inclusion criteria, were posted on the bulletin board in each local clinic's reception area. The inclusion criteria were: 1 ) mother $\geq 18$ years; 2 ) infant aged between 6 months and 18 months at the time of survey completion; 3) living in Beijing and breastfeeding their infant (exclusively or partially) for some or all of the lockdown period (during 26th January to 31st July 2020); 4) mother had no complications or other chronic disease during the pregnancy or postnatal period. Eligible mothers were invited to complete a one-time, anonymous questionnaire, which consisted of 48 questions and took approximately $15-20 \mathrm{~min}$ to complete. The questionnaire was provided both online and in a paper version and mothers could choose which one they wanted to complete. The online version was supported by the WenjuanNet (https://wenjuan.net/s/NVveyew/), which can create online questionnaire with a large number of templates 
provided. A QR code was generated when the online survey started. Eligible mothers who were interested in participation could begin the survey on their smart phone by scanning the QR code, or by clicking the invitation link.

Respondents were informed that their participation was voluntary and their consent to this study was implied by completion of the questionnaire. The first page of the survey provided information about the study.
Mothers could complete the questionnaire during their waiting time in the clinic, and a trained nurse was available to assist the mothers in completing the questionnaire. We finally retrieved 1807 eligible mother's information. The response rate for each surveyed question ranged 90-100\% (as shown in Tables 1, 2 and 3). Two investigators imported the raw data into SPSS separately. If there were discrepancies, they both would double-check the original questionnaire and correct the potential errors.

Table 1 Background characteristics of mothers who completed the survey (mean \pm SD / $n(\%)$ )

\begin{tabular}{|c|c|c|c|c|c|}
\hline Mean \pm SD / N(\%) & N (\%) & $\begin{array}{l}\text { Before } \\
(N=1231)\end{array}$ & $\begin{array}{l}\text { During } \\
(N=576)\end{array}$ & $\begin{array}{l}\text { Total } \\
(N=1807)\end{array}$ & $P$ value \\
\hline Maternal age (years) & $1771(98)$ & $31.6 \pm 4.1$ & $30.9 \pm 4.2$ & $31.4 \pm 4.2$ & 0.000 \\
\hline Maternal schooling years & $1775(98.2)$ & $15.3 \pm 2.6$ & $15.3 \pm 2.5$ & $15.3 \pm 2.6$ & 0.990 \\
\hline Maternal education & $1787(98.9)$ & & & & \\
\hline Diploma or under & & $528(43.3)$ & $255(44.8)$ & $783(43.8)$ & 0.889 \\
\hline Bachelor's degree & & $504(41.4)$ & $234(41.1)$ & $738(41.3)$ & \\
\hline Master's degree & & $168(13.8)$ & $73(12.8)$ & $241(13.5)$ & \\
\hline PhD/professional qualification & & $18(1.5)$ & $7(1.2)$ & $25(1.4)$ & \\
\hline Household income in CNY & $1791(99.1)$ & & & & \\
\hline$<20,000$ & & 431 (35.3) & $215(37.8)$ & $646(36.1)$ & 0.031 \\
\hline$<30,000$ & & $264(21.6)$ & $148(26)$ & $412(23)$ & \\
\hline$<40,000$ & & $168(13.7)$ & $62(10.9)$ & $230(12.8)$ & \\
\hline$<50,000$ & & $67(5.5)$ & $17(3)$ & $84(4.7)$ & \\
\hline$>50,000$ & & $33(2.7)$ & $17(3)$ & $50(2.8)$ & \\
\hline Other & & $15(1.2)$ & $3(0.5)$ & $18(1)$ & \\
\hline Prefer not to say & & $244(20)$ & $107(18.8)$ & $351(19.6)$ & \\
\hline Maternal social class & $1713(94.8)$ & & & & \\
\hline IV \& V & & $358(30.7)$ & $172(31.5)$ & $530(30.9)$ & 0.762 \\
\hline III & & $375(32.1)$ & $181(33.2)$ & $556(32.5)$ & \\
\hline$|\&| \mid$ & & $434(37.2)$ & $193(35.3)$ & $627(36.6)$ & \\
\hline Infant gestation (weeks) & $1783(98.7)$ & $38.8 \pm 1.5$ & $38.8 \pm 1.4$ & $38.8 \pm 1.5$ & 0.740 \\
\hline Infant gender & $1803(99.8)$ & & & & \\
\hline Male & & $645(52.5)$ & $279(48.6)$ & $924(51.2)$ & 0.125 \\
\hline Female & & $584(47.5)$ & $295(51.4)$ & $879(48.8)$ & \\
\hline Infant age (months) & $1807(100)$ & $10.9 \pm 3.1$ & $6.6 \pm 1.4$ & $9.5 \pm 3.3$ & 0.000 \\
\hline Total No. of children in household & $1794(99.3)$ & & & & \\
\hline more than one child & & $454(37.1)$ & $211(37)$ & $665(37.1)$ & 0.945 \\
\hline only one child & & $769(62.9)$ & $360(63)$ & $1129(62.9)$ & \\
\hline Current accommodation & $1631(90.3)$ & & & & \\
\hline Bungalow & & $5(0.5)$ & $4(0.8)$ & $9(0.6)$ & 0.000 \\
\hline T-apartment without garden & & $112(10.2)$ & $124(23.4)$ & $236(14.5)$ & \\
\hline T-apartment with garden & & $259(23.5)$ & $100(18.9)$ & $359(22)$ & \\
\hline B-apartment without garden & & $162(14.7)$ & $66(12.5)$ & $228(14)$ & \\
\hline B-apartment with garden & & $538(48.8)$ & $231(43.7)$ & $769(47.1)$ & \\
\hline Villa & & $26(2.4)$ & $4(0.8)$ & $30(1.8)$ & \\
\hline
\end{tabular}

Notes: ${ }^{*} P<0.05 . \mathrm{SD}=$ standard deviation

a $\mathrm{T}$-apartment is a type of apartment building with around ten residents living on each floor

${ }^{b}$ B-apartment is a type of apartment with less than three residents living on each floor

Maternal age refers to the age at delivery 
Table 2 Maternal birth experience and COVID impact during lockdown, according to whether they delivered before or during the lockdown

\begin{tabular}{|c|c|c|c|c|c|}
\hline$N(\%)$ & $N(\%)$ & $\begin{array}{l}\text { Before } \\
(N=1231)\end{array}$ & $\begin{array}{l}\text { During } \\
(N=576)\end{array}$ & $\begin{array}{l}\text { Total } \\
(N=1807)\end{array}$ & $P$ value \\
\hline Living status during the lockdown & $1774(98.2)$ & & & & \\
\hline With husband & & $409(33.7)$ & $221(39.5)$ & $630(35.5)$ & 0.168 \\
\hline With husband \& parents & & $310(25.5)$ & $136(24.3)$ & $446(25.1)$ & \\
\hline With husband \& parents-in-law & & $481(39.6)$ & $199(35.5)$ & $680(38.3)$ & \\
\hline Alone & & $9(0.7)$ & $2(0.4)$ & $11(0.6)$ & \\
\hline Other & & $5(0.4)$ & $2(0.4)$ & $7(0.4)$ & \\
\hline COVID impact on purchasing daily necessities & $1792(99.2)$ & & & & \\
\hline Great & & $106(8.7)$ & $57(10)$ & $163(9.1)$ & 0.014 \\
\hline Moderate & & $433(35.5)$ & $232(40.6)$ & $665(37.1)$ & \\
\hline Little & & $375(30.7)$ & $177(30.9)$ & $552(30.8)$ & \\
\hline No & & $306(25.1)$ & $106(18.5)$ & $412(23)$ & \\
\hline COVID impact on household income & $1797(99.4)$ & & & & \\
\hline Great & & $144(11.8)$ & $75(13.1)$ & $219(12.2)$ & 0.013 \\
\hline Moderate & & $466(38.1)$ & $246(42.9)$ & $712(39.6)$ & \\
\hline Little & & $297(24.3)$ & $143(25)$ & $440(24.5)$ & \\
\hline No & & $317(25.9)$ & $109(19)$ & $426(23.7)$ & \\
\hline COVID impact on birth location & $1788(98.9)$ & & & & \\
\hline Yes & & $58(4.8)$ & $61(10.7)$ & $119(6.7)$ & 0.000 \\
\hline No & & $1161(95.2)$ & $508(89.3)$ & $1669(93.3)$ & \\
\hline Birth location & $1806(99.9)$ & & & & \\
\hline National hospital & & 1177 (95.7) & $552(95.8)$ & $1729(95.7)$ & 0.592 \\
\hline Private hospital & & $49(4)$ & $24(4.2)$ & $73(4)$ & \\
\hline Oversea hospital & & $3(0.2)$ & $0(0)$ & $3(0.2)$ & \\
\hline Home & & $1(0.1)$ & $0(0)$ & $1(0.1)$ & \\
\hline Mode of delivery & $1802(99.7)$ & & & & \\
\hline Vaginal & & $744(60.6)$ & $359(62.4)$ & $1103(61.2)$ & 0.656 \\
\hline Vaginal induced & & $38(3.1)$ & $22(3.8)$ & $60(3.3)$ & \\
\hline Planned caesarean & & $300(24.4)$ & $133(23.1)$ & $433(24)$ & \\
\hline Emergency caesarean & & $145(11.8)$ & $61(10.6)$ & $206(11.4)$ & \\
\hline Skin-to-skin contact after delivery & $1804(99.8)$ & & & & \\
\hline Yes & & $1184(96.3)$ & $555(96.7)$ & 1739 (96.4) & 0.648 \\
\hline No & & $46(3.7)$ & $19(3.3)$ & 65 (3.6) & \\
\hline
\end{tabular}

Note: ${ }^{*} \mathrm{P}<0.05$

Data from the online questionnaire were automatically exported to the SPSS.

During the survey design, we calculated the expected sample size using the following function [17]:

$$
n=\left[\frac{\left.Z_{\alpha} \sqrt{\left(p_{1}+p_{2}\right)\left(1-p_{1}+1-p_{2}\right) / 2}+z_{\beta}\right) \sqrt{p_{1}\left(1-p_{1}\right)+p_{2}\left(1-p_{2}\right)}}{p_{1}-p_{2}}\right]^{2}
$$

Based on previous literature, we estimated that the breast-feeding rate for first 6 months was $50 \%$ [18] and the estimated rate difference was $8 \%$ (p2 to be $58 \%$ ), with statistical level $\alpha=0.05$ and power $=0.8(1-\beta)$, two-sided test, the total sample size should be at least 1800 .

Ethical approval was obtained from the Beijing Children's Hospital Research Ethics committee (2020-Z-102).

\section{Content of the survey}

The questionnaire was adapted from one intended for use in the UK, namely the COVID-19 New Mum Study $[9,19]$ with translation into Chinese. A repeated forward-backward translation procedure was adopted. The translated Chinese version was produced by a native 
Table 3 Infant feeding practice, COVID impacts, and relevant feeding supports of subjects, according to whether they delivered before or during the lockdown

\begin{tabular}{|c|c|c|c|c|c|}
\hline $\mathrm{N}(\%)$ & $N(\%)$ & $\begin{array}{l}\text { Before } \\
(N=1231)\end{array}$ & $\begin{array}{l}\text { During } \\
(N=576)\end{array}$ & $\begin{array}{l}\text { Total } \\
(N=1807)\end{array}$ & $P$ value \\
\hline Feeding intention before delivery & 1800 (99.6) & & & & \\
\hline Exclusive breastfeeding & & $1014(82.8)$ & $485(84.2)$ & 1499 (83.3) & 0.660 \\
\hline Formula feeding & & $31(2.5)$ & $13(2.3)$ & $44(2.4)$ & \\
\hline Mixed feeding & & $120(9.8)$ & $47(8.2)$ & $167(9.3)$ & \\
\hline No plans & & $59(4.8)$ & $31(5.4)$ & $90(5)$ & \\
\hline Infant feeding at 6-month & 1800 (99.6) & & & & \\
\hline Mostly breastfeeding & & $746(60.9)$ & $392(68.1)$ & $1138(63.2)$ & 0.014 \\
\hline Formula feeding & & $79(6.5)$ & $32(5.6)$ & $111(6.2)$ & \\
\hline Mixed feeding & & $399(32.6)$ & $152(26.4)$ & $551(30.6)$ & \\
\hline Change in feeding intention and actual feeding for 6 month & $1806(99.9)$ & & & & 0.013 \\
\hline Yes, change & & $366(29.8)$ & $139(24.1)$ & $505(28)$ & \\
\hline No change & & $864(70.2)$ & $437(75.9)$ & $1301(72)$ & \\
\hline Current infant feeding & $1806(99.9)$ & & & & \\
\hline Mostly breastfeeding & & $88(7.2)$ & $210(36.5)$ & $298(16.5)$ & 0.000 \\
\hline Breastfeeding + solids & & $397(32.3)$ & $150(26)$ & $547(30.3)$ & \\
\hline Formula feeding & & $38(3.1)$ & $15(2.6)$ & $53(2.9)$ & \\
\hline Formula feeding plus solid & & $261(21.2)$ & $43(7.5)$ & $304(16.8)$ & \\
\hline Mixed milk feeding & & $44(3.6)$ & $42(7.3)$ & $86(4.8)$ & \\
\hline Mixed feeding plus solids & & $402(32.7)$ & $116(20.1)$ & $518(28.7)$ & \\
\hline COVID impact on original infant feeding & $1796(99.4)$ & & & & \\
\hline Great & & $66(5.4)$ & $30(5.3)$ & $96(5.3)$ & 0.036 \\
\hline Moderate & & $230(18.8)$ & $140(24.5)$ & $370(20.6)$ & \\
\hline Little & & $246(20.1)$ & $115(20.1)$ & $361(20.1)$ & \\
\hline No & & $683(55.8)$ & $286(50.1)$ & $969(54)$ & \\
\hline Received enough help with BF from professionals & $1804(99.8)$ & & & & \\
\hline Yes & & $1170(95.3)$ & $557(96.7)$ & $1727(95.7)$ & 0.163 \\
\hline No & & $58(4.7)$ & $19(3.3)$ & $77(4.3)$ & \\
\hline Received enough help with BF from family & $1804(99.8)$ & & & & \\
\hline Yes & & $1164(94.8)$ & $553(96)$ & $1717(95.2)$ & 0.260 \\
\hline No & & $64(5.2)$ & $23(4)$ & $87(4.8)$ & \\
\hline Enough support with own health & $1693(93.7)$ & & & & \\
\hline Yes & & $1048(90.5)$ & $488(91.2)$ & $1536(90.7)$ & 0.638 \\
\hline No & & $110(9.5)$ & $47(8.8)$ & $157(9.3)$ & \\
\hline Contact with a health professional (per week) & $1783(98.7)$ & & & & \\
\hline Never & & $756(62.3)$ & $347(61)$ & $1103(61.9)$ & 0.010 \\
\hline $1-3$ times & & $371(30.6)$ & $166(29.2)$ & $537(30.1)$ & \\
\hline 4-5 times & & $29(2.4)$ & $31(5.4)$ & $60(3.4)$ & \\
\hline Daily or more & & $58(4.8)$ & $25(4.4)$ & $83(4.7)$ & \\
\hline Chat in a breastfeeding support group (per week) & $1785(98.8)$ & & & & \\
\hline Never & & $539(44.4)$ & $228(40)$ & $767(43)$ & 0.081 \\
\hline $1-3$ times & & $476(39.2)$ & $231(40.5)$ & 707 (39.6) & \\
\hline $4-5$ times & & $93(7.7)$ & $41(7.2)$ & $134(7.5)$ & \\
\hline Daily or more & & $107(8.8)$ & $70(12.3)$ & $177(9.9)$ & \\
\hline \multicolumn{6}{|l|}{ Who supports infant feeding? (multiple choices allowed) } \\
\hline Partner & $1791(99.1)$ & $774(63.5)$ & $392(68.5)$ & $1166(65.1)$ & 0.037 \\
\hline Parent & $1791(99.1)$ & $691(56.7)$ & $301(52.6)$ & $992(55.4)$ & 0.107 \\
\hline Parent In-laws & $1791(99.1)$ & $557(45.7)$ & $260(45.5)$ & $817(45.6)$ & 0.925 \\
\hline
\end{tabular}


Table 3 (continued)

\begin{tabular}{|c|c|c|c|c|c|}
\hline $\mathrm{N}(\%)$ & $N(\%)$ & $\begin{array}{l}\text { Before } \\
(N=1231)\end{array}$ & $\begin{array}{l}\text { During } \\
(N=576)\end{array}$ & $\begin{array}{l}\text { Total } \\
(N=1807)\end{array}$ & $P$ value \\
\hline Health professional & $1791(99.1)$ & $551(45.2)$ & $207(36.2)$ & $758(42.3)$ & 0.000 \\
\hline Friend/relative & $1791(99.1)$ & $356(29.2)$ & $125(21.9)$ & $481(26.9)$ & 0.001 \\
\hline Breastfeeding group & $1791(99.1)$ & $386(31.7)$ & $164(28.7)$ & $550(30.7)$ & 0.200 \\
\hline Online support & $1791(99.1)$ & $243(19.9)$ & $82(14.3)$ & $325(18.1)$ & 0.004 \\
\hline Other & $1791(99.1)$ & $14(1.1)$ & $2(0.3)$ & $16(0.9)$ & 0.094 \\
\hline No support & $1791(99.1)$ & $39(3.2)$ & $7(1.2)$ & $46(2.6)$ & 0.014 \\
\hline
\end{tabular}

Notes: ${ }^{*} \mathrm{P}<0.05$. For multiple choices question, we compared each choice for DL \& BL using the Chi-square. For single choice question we used the Chi-square and Mann-Whitney test for all categories

Chinese speaker on the team and refined by a $\mathrm{BCH}$ paediatrician with clinical experience of infant feeding. Then the Chinese version was translated back into English and compared to the original English version. The survey comprises of four sections: 1) demographic characteristics of the participants; 2) maternal birth experiences and infant feeding; for the evaluation of infant feeding method, mothers were asked about the method of milk feeding used during the first 6 months ("what is the source of milk you used for feeding for the first six months after birth?"). The response options were exclusive breastfeeding, mixed feeding or formula-feeding. However, given that a strict definition of exclusive breastfeeding was not provided, and the question did not ask specifically about complementary foods, the exclusive breastfeeding category was renamed as 'mostly breastfeeding' (MBF) for the analyses, reflecting the majority source of the infant's milk intake during this period.; 3) Maternal daily life and supports availables; and 4) the impact of COVID-19 on maternal mental health and life patterns. Detailed content is outlined in the appendix and can also be found in a previous publication using the same questionnaire $[9,20]$.

\section{Statistical analyses}

For aim (1), we conducted descriptive analysis for mother's background characteristics. For continuous variables (maternal age, maternal schooling year, infant gestational age and infant age), the mean (standard deviation, SD) was estimated for the eligible sample and for women who delivered before/during lockdown period, and independent $\mathrm{t}$-test was used to compare the groups. For binary or categorical variables, frequency tables and cross tabs were applied to estimate the $\mathrm{N}(\%)$ in each cell with chisquare test used to estimate the statistical difference.

For aim (2), to compare the distribution of mother's birth experiences, infant feeding practices, COVID lockdown impact and support received among mothers delivered before/during lockdown, we also applied mainly cross tabs and chi-square to test the statistical difference.

Finally for aim (3), in order to investigate the determinants of maternal feeding practice for the first 6-months (breastfeeding or other feeding approaches) and to estimate whether lockdown had an impact on maternal feeding practice after controlling for other determinants, we first conducted univariate analysis (independent t-test or chi-square test) to explore the potential determinants (maternal SES backgrounds, received breastfeeding support, and delivery status) for maternal feeding practice. Then we applied logistic regression to estimate the relationship between maternal feeding practice during the first 6 months and delivered before/during the lockdown, with adjustment for covariates including maternal SES backgrounds and living conditions, delivery conditions, health and infant feeding support and COVID lockdown impact. Logistic regression was performed based on the Directed Acyclic Graph (DAG, eFigure 1). Adjusted odds ratio (OR) and $95 \%$ confidence intervals (CI) are presented, and $P<0.05$ was considered as statistically significant. All statistical analyses were conducted using SPSS version 22.0 (IBM., Armonk, NY, USA).

\section{Results}

\section{Demographic characteristics of the participants}

From 1st August to 31st October 2020, 1807 mothers completed the questionnaire. All participants were married. At the time of survey completion, mean maternal age at delivery was $31.4 \pm 4.2$ years, and mean infant age $9.5 \pm 3.3$ months (range 1-18 months). The background characteristics are shown in Table 1 . There were 576 (31.9\%) and 1231 (68.1\%) mothers who gave birth during the lockdown (DL) and before the lockdown (BL), respectively. Maternal education and infant gestation did not differ between the BL and DL groups. Infant age was higher as expected in BL (10.9 \pm 3.1 months) than in DL 
$(6.6 \pm 1.4$ months $)(p<0.001)$ due to the experimental design of the study. Mothers in the BL group were also older at delivery than those in the DL group $(p<0.001)$ and more were in the lowest category for education and household income compared to the DL group $(p<0.05)$.

\section{Birth experiences and daily life}

For DL and BL mothers, 62.4 and $60.6 \%$ were vaginally delivered and the post-birth skin-to-skin contact was 96.7 and $96.3 \%$ respectively; with no significant differences between groups. Over $95 \%$ of the women delivered at a national hospital. Notably, significantly more mothers in DL groups reported that the COVID-19 had impact on their birth location, compare to BL mothers $(10.7 \%$ vs. $4.8 \%, p<0.001)$. Moreover, compared to $\mathrm{BL}$ mothers, significantly more women in the DL group considered COVID-19 to have had a moderate-to-high impact on their household income ( $49.9 \%$ vs. $56 \%, p=0.013)$ and on the convenience of purchasing daily necessities $(44.2 \% \mathrm{vs}$. $50.6 \%, p=0.014$ ) (Table 2).

\section{Infant feeding during the lockdown}

Infant feeding intentions did not differ between DL and BL groups (Table 3). Among DL women, 29.8\% reported a moderate-to-high impact of COVID-19 on their infant feeding practice, compared to $24.2 \%$ in the $\mathrm{BL}$ group $(p=0.036)$. In the DL group, significantly more mothers reported 6-month MBF compared to the BL mothers $(68.1 \%$ vs. $60.9 \%, p=0.014)$. As expected, given the younger age of the infants of DL mothers, a significantly higher proportion of mothers were MBF (36.5\%) compared to BL mothers (7.2\%) at the time of completing the questionnaire, while a greater proportion of older infants born BL also consumed solid foods (Table 3).

\section{Infant feeding support during the lockdown}

Overall, more than $90 \%$ of mothers in both BL and DL groups reported they had received enough supports during the lockdown, by answering "yes" to the question "Did you received enough help with breastfeeding from professionals/family?" and "Did you received enough support with your own health?" (Table 3), suggesting that mothers who had given birth during the pandemic may have received same overall support than those who had given birth before. The main reported sources of infant feeding support ('Where do you get support with infant feeding?') were the partner (65.1\%), followed by parents (55.4\%), and parents-inlaw (45.6\%), health professionals (42.3\%), breastfeeding support groups (30.7\%), friends and family (26.9\%), and online support (18.1\%). A significantly higher proportion of DL mothers reported having received support from their partner compared to the BL group, whilst a larger proportion of BL mothers received support from health professional, friends and relatives, online support sources or reported no support during the pandemic (Table 3).

No difference was found between BL and DL mothers regarding the frequency of participating in a breastfeeding support group. Significantly more DL women reported having contacted health professionals more than four times a week (19.5\%), compared to those from the BL group (16.5\%).

\section{Predictors of infant feeding during the first 6-months}

Results of the logistic regression show that MBF during the first 6 months was positively predicted by delivery DL versus BL (OR1.43, 95\%CI 1.08, 1.90). Positive predictors of MBF (Table 4) also included younger maternal age, infant feeding discussion in an online support group more than four times a week, and receiving support from friends or relatives versus no support.

\section{Comments}

\section{Summary of the results}

Our findings show that, despite the difficulties imposed by the pandemic, hospital facilities in Beijing continued to implement measures based on WHO guidelines [8, $21,22]$. No significant differences were found in maternal delivery method, postnatal skin-to-skin contact, or breastfeeding supports between $\mathrm{BL}$ and DL mothers. However, significant differences were found in perceived lockdown impact on household income, convenience of buying daily necessities, changes of birth location, feeding plan, and current feeding methods between BL and DL mothers. The MBF at 6 -months was predicted by delivery during the lockdown period, younger maternal age, getting support from friends or relatives, and discussing health issues in online groups more than four times a week.

\section{Maternal birth experience, infant feeding practice and support}

A significantly higher number of mothers in DL group reported their intended birth location had been affected by the pandemic, reflecting mothers who originally planned to give birth at a private hospital but were changed to a public hospital or vice versa. Some may have chosen a private hospital in order to reduce contact with others, whereas some mothers may have believed that a public hospital could provide better treatment than a private maternity hospital should they become infected; however, we did not record this information as part of the survey.

At 6 months, significantly more DL mothers were mostly breastfeeding compared to BL mothers, 
Table 4 Univariate and multivariable logistic regression for determinants of whether or not MBF for the first 6 months

\begin{tabular}{|c|c|c|c|c|c|c|c|c|}
\hline \multirow[t]{3}{*}{ 6-month mostly breastfeeding rate } & \multirow[t]{3}{*}{$\mathrm{N}(\%)$} & \multicolumn{3}{|c|}{ Univariate analysis } & \multirow{2}{*}{\multicolumn{4}{|c|}{$\begin{array}{l}\text { Multivariable Logistic Regression } \\
95 \% \text { CI for OR }\end{array}$}} \\
\hline & & \multirow{2}{*}{$\begin{array}{l}\text { Non-MBF } \\
(N=662)\end{array}$} & \multirow{2}{*}{$\begin{array}{l}\text { MBF } \\
(N=1138)\end{array}$} & \multirow{2}{*}{$\begin{array}{l}P \text { value } \\
(T=1800)\end{array}$} & & & & \\
\hline & & & & & OR & Lower & Upper & $P$ value \\
\hline Infant born before/during lockdown? & $1800(100)$ & & & 0.003 & & & & \\
\hline born before lockdown & & $479(39.1)$ & $746(60.9)$ & & ref & & & \\
\hline born during lockdown & & $184(31.9)$ & $392(68.1)$ & & 1.43 & 1.08 & 1.90 & 0.012 \\
\hline 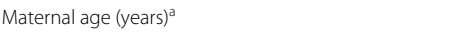 & $1764(98)$ & $31.8 \pm 4.3$ & $31.2 \pm 4.1$ & 0.000 & 0.96 & 0.93 & 0.99 & 0.011 \\
\hline Total years of maternal full-time education & $1769(98.3)$ & $15.4 \pm 2.5$ & $15.3 \pm 2.6$ & 0.620 & 1.02 & 0.96 & 1.07 & 0.618 \\
\hline Household annual income in CNY & $1417(78.7)$ & & & 0.781 & & & & \\
\hline$<200,000$ & & $239(37.2)$ & $404(62.8)$ & & ref & & & \\
\hline $200,000-300,000$ & & $161(39.2)$ & $250(60.8)$ & & 0.94 & 0.69 & 1.29 & 0.700 \\
\hline$>300,000$ & & $135(37.2)$ & $228(62.8)$ & & 1.36 & 0.96 & 1.93 & 0.089 \\
\hline Maternal social class ${ }^{b}$ & $1706(94.8)$ & & & 0.233 & & & & \\
\hline IV \& V & & $185(35.1)$ & $342(64.9)$ & & ref & & & \\
\hline III & & $197(35.6)$ & $357(64.4)$ & & 1.08 & 0.76 & 1.53 & 0.666 \\
\hline I\&\| & & $247(39.5)$ & $379(60.5)$ & & 0.77 & 0.55 & 1.07 & 0.113 \\
\hline Building living ${ }^{c}$ & $1627(90.4)$ & & & 0.204 & & & & \\
\hline Bungalow & & $5(55.6)$ & $4(44.4)$ & & ref & & & \\
\hline Tapartment without garden & & $79(33.5)$ & $157(66.5)$ & & 3.82 & 0.66 & 22.13 & 0.134 \\
\hline Tapartment with garden & & $119(33.2)$ & $239(66.8)$ & & 4.75 & 0.83 & 27.16 & 0.080 \\
\hline B apartment no garden & & $83(36.4)$ & $145(63.6)$ & & 3.67 & 0.64 & 21.21 & 0.146 \\
\hline B apartment with garden & & $302(39.4)$ & $465(60.6)$ & & 2.82 & 0.50 & 15.84 & 0.240 \\
\hline Villa with garden & & $9(30)$ & $21(70)$ & & 3.53 & 0.50 & 25.18 & 0.208 \\
\hline whether had support from families/friends after birth & $1797(99.8)$ & & & 0.360 & & & & \\
\hline No & & $36(41.4)$ & $51(58.6)$ & & ref & & & \\
\hline Yes & & $625(36.5)$ & $1086(63.5)$ & & 1.95 & 1.06 & 3.59 & 0.032 \\
\hline Enough infant feeding support? & $1784(99.1)$ & & & 0.053 & & & & \\
\hline not enough & & $23(50)$ & $23(50)$ & & ref & & & \\
\hline enough & & $628(36.1)$ & $1111(63.9)$ & & 1.44 & 0.69 & 3.03 & 0.334 \\
\hline Enough support with own health? & $1686(93.7)$ & & & 0.060 & & & & \\
\hline not enough & & $68(43.3)$ & $89(56.7)$ & & ref & & & \\
\hline enough & & $548(35.8)$ & $982(64.2)$ & & 1.42 & 0.90 & 2.24 & 0.135 \\
\hline Contact with a health professional (/week) & $1776(98.7)$ & & & 0.050 & & & & \\
\hline Never & & $428(38.9)$ & $673(61.1)$ & & ref & & & \\
\hline $1-3$ times/week & & $183(34.3)$ & $351(65.7)$ & & 1.05 & 0.77 & 1.43 & 0.759 \\
\hline $4+$ times/week & & $43(30.3)$ & $99(69.7)$ & & 1.12 & 0.65 & 1.94 & 0.684 \\
\hline Discuss health issues in mothers' group online (/week) & $1778(98.8)$ & & & 0.040 & & & & \\
\hline Never & & $305(39.9)$ & $459(60.1)$ & & ref & & & \\
\hline 1-3 times/week & & $251(35.6)$ & $455(64.4)$ & & 1.23 & 0.91 & 1.68 & 0.177 \\
\hline $4+$ times/week & & $99(32)$ & $210(68)$ & & 1.66 & 1.09 & 2.53 & 0.019 \\
\hline Mode of delivery & $1796(99.8)$ & & & 0.000 & & & & \\
\hline Vaginal & & $387(33.4)$ & $772(66.6)$ & & ref & & & \\
\hline Caesarean & & $275(43.1)$ & $363(56.9)$ & & 0.77 & 0.59 & 1.01 & 0.059 \\
\hline whether COVID impacted birth location & $1782(99)$ & & & 0.584 & & & & \\
\hline Yes & & $41(34.5)$ & $78(65.5)$ & & ref & & & \\
\hline No & & $615(37)$ & 1049 (63) & & 1.67 & 0.96 & 2.90 & 0.067 \\
\hline COVID impact on purchasing daily necessities & $1785(99.2)$ & & & 0.355 & & & & \\
\hline Moderate-great impact & & $294(35.6)$ & $532(64.4)$ & & ref & & & \\
\hline Little or no impact & & $362(37.7)$ & $598(62.3)$ & & 0.80 & 0.61 & 1.04 & 0.096 \\
\hline
\end{tabular}

Notes: MBF Mostly breastfeeding, $\mathrm{Cl}$ confidence interval, $D L$ mothers who delivered during the lockdown, $B L$ mothers who delivered before the lockdown. Factors controlled for included maternal socio-economic status (education, income, social class), living conditions, cohabitation with family members ("Who did you live with during the lockdown?"), gestational age, health advice-seeking behaviour ("Frequency of discussing with health professionals/chatting in breastfeeding support group"), support ("Did you receive enough support from health professionals/family members?"), and the impact of the COVID-19 lockdown on maternal daily life a Younger maternal age as reference

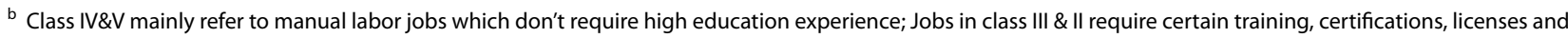
degree to qualify; Jobs in class I involve professional careers which require advanced degree, high-end skills or expertise

c T-apartment is a type of apartment building with around ten residents live in each floor; and B-apartment is a type of apartment with less than three residents live in each floor 
suggesting that the lockdown measures may have indirectly promoted breastfeeding, perhaps by allowing mothers to spend more time with their babies, or feeling less pressure due to fewer visitors during the lockdown [16]. A similar finding was reported in the COVID-19 New Mum Study in the UK [9], where 13\% of women reported changes to feeding method due to the lockdown; a higher frequency and longer duration of breastfeeding was observed in 30 and $17 \%$ women who were breastfeeding during the pandemic, reflecting more time spent at home as well as a greater contribution to childcare from themselves and partners during the lockdown period. However, contrasting findings were observed in another cross-sectional study, which reported a slight decrease in breastfeeding in 903 mothers with infants ages $0-12$ months during the COVID-19 lockdown in Thailand [23]. Thus, the impact of lockdown measures on infant feeding might differ depending on access to support and the particular circumstances experienced by individual mothers. Indeed, our results suggest that although DL and BL mothers reported to have received sufficient support in general, significant differences were observed between groups regarding the exact person who provided the main supports. Nevertheless, both peer and professional support would be important for the success of breastfeeding [24].

Research on breastfeeding support has consistently identified the husband or partner as an important source of support for women, with influence on four aspects in particular: breastfeeding decisions, assistance at first feeding, assistance during breastfeeding, and risk factors for bottle feeding [13]. Our study found that women from both groups considered their partner as the greatest source of support for infant feeding, consistent with the UK New Mum study [9] . Compared to BL mothers, DL mothers received even more support from their partners, possibly reflecting the "work from home" measures in place during the lockdown, which increased the time husbands could spend with their wives. As suggested by Vazquez et al. [9], husbands who contribute more to childcare during this exceptional period may represent a valuable source of support for women, especially for those with limited access to friends or relatives who would provide this support under normal circumstances. Hence. Partner's supports should be emphasized in breastfeeding education activities.

According to Chinese tradition, mothers normally adhere to a month long "confinement period" after birth. Although an increasing number of mothers use a confinement centre where they can receive care and professional support, most centres were closed during the lockdown period; this may have led to differences in the confinement experience for mothers in the DL and $\mathrm{BL}$ groups. However, a recent study found that the 1- and 6-month EBF rate did not differ between mothers who chose a confinement centre and mothers who stayed at home ( $37 \%$ vs. $42 \%, p=0.5)$ [25]. Whilst more studies are needed to confirm this finding, we suggest that mothers in the DL group may have received more support from their partner, so the total support received by the DL and BL mothers might have been the same.

\section{Predictors of infant feeding during the lockdown}

Mothers who delivered during the lockdown were more likely to mostly breastfeed their infant during the first 6 months. This may reflect the increased time available for both mothers and their partners to spend with the infant and the breastfeeding support provided during the lockdown. Higher rates of MBF were also observed among mothers who delivered at a younger age. This result is consistent with a previous study in Zhejiang, China, which indicated that EBF was positively related to younger maternal age [11]. However, this contrasts with studies in many high-income countries which indicated that older maternal age was associated with a higher rate of breastfeeding initiation and the duration of EBF [14, 26, 27]. This may reflect social and cultural factors, and emphasizes the importance of considering these factors when developing health policy to promote breastfeeding. In recent years, EBF has become a "new fashion" in several modern cities in China, including Beijing, Shanghai, and some large cities in Zhejiang province, possibly reflecting the increasing number of Chinese female celebrities sharing their EBF experiences and healthy mother-infant relationships through vlogs or posts. Young women from these cities are keen to follow this new fashion and are very proud if they successfully EBF to 6 months. Such kind of "celebrity effects" can be suggested in future breastfeeding promotion campaigns. Additionally, our results suggest that discussing infant feeding in a feeding support group more than four times a week, getting support from friends or relatives compared to no support positively predicted MBF in the first 6-months, reflecting the vital role of infant feeding support in promoting breastfeeding for mothers.

It worth to notice that for the feeding method at 6-months, we re-coded the original answer "EBF" to "MBF" for the question "what is the source of milk you used for feeding for the first six months after birth?", considering we did not provide a specific definition of EBF and, whilst nurses were available to help mothers if they had any queries, some mothers may not have asked for clarification. Moreover, the question in the survey referred to milk feeding but did not specifically ask about the use of complementary foods before 6 months. However, the 6 months MBF rates in the survey population 
still appear relatively high compared to previous studies in China (60.6 and 71.6\% mothers in the BL and DL group) [28]. This might reflect the inclusion criteria for the study which specified that participants must have breastfed their infant during the lockdown. Besides, rates of EBF vary across provinces and cities in China. According to the data published in 2013, the EBF rate for 14,539 children from 30 provinces in China was $20.8 \%$ [29], whilst a large cohort study conducted in Zhejiang province involving 42,550 children reported a 3 -to 5 month EBF rate of $51.3 \%$ for girls and $46.8 \%$ for boys [28]. However, in that cohort, EBF $\geq 6$ months was defined as self-reported EBF at each of the 3 clinical visits (1, 3, and 6 months), thus the EBF rate may have been over-estimated.

\section{Strengths and limitations}

To our knowledge, this is the first cross-sectional study investigating infant feeding practices and potential predictors of infant feeding during the COVID-19 pandemic in China. Using an adapted version of questionnaire that has been used in the COVID-19 New Mum Study $[9,19]$ in the UK makes the results comparable between countries. However, the study has some limitations. Firstly, although Beijing is a metropolitan city with 20 million residents from different regions of China [30], study participants are not representative of all new mothers in China; the significantly higher gross domestic product per capita in Beijing results in a higher level of family income and education of the population [31], which may partly explain the higher MBF rate in this study [32]. However, considering that the duration of lockdown and the type of lockdown measures varied between provinces and cities in China, it would have been difficult to compare the infant feeding outcomes between cities even in a national survey. Secondly, there may be recall bias since at the time of survey completion lockdown measures had just ended in Beijing. Moreover, the questionnaire used in the present study was originally designed by our research team at the very early stage of the COVID-19 pandemic, hence, there could be potential confounding factors that did not been identified and included in the questionnaire beforehand. Last but not least, we did not clearly define EBF in our questionnaire so; consequently, we relabeled this variable as 'mostly breastfeeding (MBF)'.

\section{Conclusions}

Our findings show that the pandemic and lockdown measures in Beijing affected mothers' planned birth location and breastfeeding intention. However, despite difficulties imposed by the pandemic, feeding support was generally well preserved for mothers who delivered a baby during the lockdown, reflected in high rates of mostly breastfeeding in the first 6 months. This highlights the importance of breastfeeding support both from peers and from professionals during public health emergencies. More importantly, our results suggest that by allowing parents to spend more time to contribute to childcare during the lockdown period, the breastfeeding may be promoted. This may be important not only during public health emergencies but also under normal circumstances.

\section{Abbreviations \\ COVID-19: coronavirus disease 2019; SARS-CoV-2: severe acute respiratory syndrome coronavirus 2; DL: during the lockdown; BL: before the lockdown; MBF: mostly breastfeeding; EBF: exclusive breastfeeding; DAG: directed acyclic graphs; SD: standard deviation; OR: odds ratio; Cl: confidence intervals.}

\section{Supplementary Information}

The online version contains supplementary material available at https://doi. org/10.1186/s12887-022-03155-y.

Additional file 1: eFigure 1. Directed acyclic graph of the factors related to infant feeding at first 6 months of birth.

Additional file 2 .

\section{Acknowledgements}

Not applicable.

\section{Accordance}

The study has been performed in accordance with the Declaration of Helsinki. We confirm that all methods in this study were performed in accordance with the STROBE checklist. Ethical Approval was obtained from the Beijing Children's Hospital Research Ethics Committee (reference number: 2020-Z-102).

\section{Authors' contributions}

All authors contributed to the design and planning of the study. ZW collected the data, JY and MG drafted the manuscript. MG contributed to the data analysis. MF and JW refined the manuscript. All authors read and approved the final manuscript.

Funding

No funding received.

\section{Availability of data and materials}

The datasets used and/or analysed during the current study are available from the corresponding author on reasonable request.

\section{Declarations}

Ethics approval and consent to participate

Ethical approval was obtained from the Beijing Children's Hospital Research Ethics Committee (reference number: 2020-Z-102). The first page of the survey provided information about the study. Written informed consent was obtained from all subjects involved in the study.

\section{Consent for publication}

Not applicable.

\section{Competing interests}

The authors declare no conflict of interest with respect to this study. Professor Mary Fewtrell receives an unrestricted donation for research on infant nutrition from Philips. The remaining authors declare no other conflicts. 


\section{Author details}

${ }^{1}$ Population, Policy \& Practice Research \& Teaching Department, UCL Institute of Child Health, University College London, London, UK. ${ }^{2}$ Department of Child Health, Capital Medical University Beijing Children's Hospital, Beijing, China.

Received: 17 July 2021 Accepted: 8 February 2022

Published online: 24 February 2022

\section{References}

1. Zhou P, Yang X-L, Wang X-G, Hu B, Zhang L, Zhang W, et al. A pneumonia outbreak associated with a new coronavirus of probable bat origin. Nature. 2020;579(7798):270-3.

2. Williams J, Namazova-Baranova L, Weber M, Vural M, Mestrovic J, Carrasco-Sanz A, et al. The importance of continuing breastfeeding during coronavirus disease-2019: in support of the World Health Organization statement on breastfeeding during the pandemic. J Pediatr. 2020;223:234

3. Dong Y, Mo X, Hu Y, Qi X, Jiang F, Jiang Z, et al. Epidemiology of COVID-19 among children in China. Pediatrics. 2020;145(6): e20200702.

4. Asadi L, Tabatabaei RS, Safinejad H, Mohammadi M. New corona virus (COVID-19) management in pregnancy and childbirth. Arch Clin Infect Dis. 2020;15(COVID-19):e102938.

5. Velavan TP, Meyer CG. The COVID-19 epidemic. Tropical Med Int Health. 2020;25(3):278

6. Amer F, Hammoud S, Farran B, Boncz I, Endrei DJ, Preparedness PH. Assessment of countries' preparedness and lockdown effectiveness in fighting COVID-19. Disas Med Publ Health Preparedness. 2021;15(2):e15-22.

7. Walker KF, O'Donoghue K, Grace N, Dorling J, Comeau JL, Li W, et al. Gynaecology: maternal transmission of SARS-COV-2 to the neonate, and possible routes for such transmission: a systematic review and critical analysis. Int J Obstetr Gynaecol. 2020;127(11):1324-36.

8. World Health Organization (WHO) Coronavirus disease (COVID-19) technical guidance: Infection prevention and control/WASH. Retrieved April. 2020:2:2020

9. Vazquez-Vazquez A, Dib S, Rougeaux E, Wells JC, Fewtrell MJA. The impact of the Covid-19 lockdown on the experiences and feeding practices of new mothers in the UK: preliminary data from the COVID-19 new mum study. Appetite. 2021;156:104985.

10. Zanardo V, Tortora D, Guerrini P, Garani G, Severino L, Soldera G, et al. Infant feeding initiation practices in the context of COVID-19 lockdown. Early Hum Dev. 2021;152:105286.

11. Qiu L, Zhao Y, Binns CW, Lee AH, Xie X. Initiation of breastfeeding and prevalence of exclusive breastfeeding at hospital discharge in urban, suburban and rural areas of Zhejiang China. Int Breastfeed J. 2009;4(1):1-7.

12. Council GOotS. The state Council's announcement on the arrangement of public holidays in 2020. Edited by General Office of the State Council PsRoC. http://www.gov.cn/zhengce/content/2020-01/27/content_54723 52.htm; 2020

13. Bar-Yam NB, Darby L. Fathers and breastfeeding: a review of the literature. J Hum Lact. 1997;13(1):45-50.

14. Jones JR, Kogan MD, Singh GK, Dee DL, Grummer-Strawn LM. Factors associated with exclusive breastfeeding in the United States. Pediatrics. 2011;128(6):1117-25.

15. Prevention CfDCa: Breastfeeding and caring for newborns. In., vol. 2021. Beijing: National Center for Immunization and Respiratory Diseases; 2021.

16. Brown A, Shenker N. Experiences of breastfeeding during COVID-19: lessons for future practical and emotional support. Mater Child Nutri. 2021;17(1):e13088.

17. Ryan TP. Sample Size determination and power: Wiley; 2013.

18. Wu H, Zhang Y, Zong X, Li H. Breastfeeding rates of children under two years old in nine cities of China from 1985 to 2015: a comparison between urban and suburban areas. Chin J Perinat Med. 2019;12(1):445-50.

19. Dib S, Rougeaux E, Vazquez-Vazquez A, Wells JCK, Fewtrell M. Maternal mental health and coping during the COVID-19 lockdown in the UK: data from the COVID-19 new mum study. Int J Gynaecol Obstet. 2020:151(3):407-14.
20. Wei Z, Gao M-Y, Fewtrell M, Wells J, Yu J-Y, Maternal mental health and well-being during the COVID-19 pandemic in Beijing, China. World J Pediatr. 2021:17(3):280-9.

21. World Health Organization. Breastfeeding and COVID-19. In., vol. World Health Organization; 2020. Online at:WHO/2019-nCoV/Sci_Brief/ breastfeeding/2020.1.

22. World Health Organization. Clinical management of COVID-19: interim guidance, 27 May 2020. World Health Organization; 2020.

23. Piankusol C, Sirikul W, Ongprasert K, Siviroj PJ, Health P. Factors affecting breastfeeding practices under lockdown during the COVID-19 pandemic in Thailand: a cross-sectional survey. Int J Environ Res Public Health. 2021;18(16):8729.

24. Schmied V, Beake S, Sheehan A, McCourt C, Dykes F. Women's perceptions and experiences of breastfeeding support: a metasynthesis. Birth. 2011;38(1):49-60

25. Foong SC, Tan ML, Foong WC, Ho JJ, Rahim FF. Comparing breastfeeding experiences between mothers spending the traditional Chinese confinement period in a confinement Centre and those staying at home: a cohort study. Int Breastfeed J. 2021;16(1):1-9.

26. Kitano N, Nomura K, Kido M, Murakami K, Ohkubo T, Ueno M, et al. Combined effects of maternal age and parity on successful initiation of exclusive breastfeeding. Prev Med Rep. 2016;3:121-6.

27. Kristiansen AL, Lande B, Øverby NC, Andersen LF. Factors associated with exclusive breast-feeding and breast-feeding in Norway. Public Health Nutr. 2010;13(12):2087-96.

28. Zheng J-S, Liu H, Li J, Chen Y, Wei C, Shen G, et al. Exclusive breastfeeding is inversely associated with risk of childhood overweight in a large Chinese cohort. J Nutr. 2014;144(9):1454-9.

29. Yang ZY, Lai JQ, Yu DM, Duan YF, Pang XH, Jiang S, et al. Breastfeeding rates in China: a cross-sectional survey and estimate of benefits of improvement. Lancet. 2016:388:47.

30. World Population Review. Beijing Population 2021. World Population Review 2021; 2021. Online at: https://worldpopulationreview.com/worldcities/beijing-population.

31. Textor C: Per capita GDP in Beijing, China 2010-2019. In. Online at: https://www.statista.com/statistics/989807/china-per-capita-gdp-beiji ng/: Statista; 2021.

32. Brand $E$, Kothari $C$, Stark MA. Factors related to breastfeeding discontinuation between hospital discharge and 2 weeks postpartum. J Perinat Educ. 2011;20(1):36-44.

\section{Publisher's Note}

Springer Nature remains neutral with regard to jurisdictional claims in published maps and institutional affiliations. 